\title{
STUDY ON RELATION BETWEEN INELASTIC AND ELASTIC DISPLACEMENT FOR VRANCEA EARTHQUAKES
}

\begin{abstract}
Adrian GUTUNOI - Assistant, Technical University of Civil Engineering, Faculty of Civil, Industrial and Agrarian Structures, e-mail: adrian.gutunoi@yahoo.com

Dan ZAMFIRESCU - Associate Professor, Technical University of Civil Engineering, Faculty of Civil, Industrial and Agrarian Structures, e-mail: dzam@utcb.ro

Abstract: At present, Romanian code for seismic design P100 is in a process of assimilation of the requirements from the equivalent European code, EN-1998-1. However, the unique characteristics of the Vrancea earthquakes require additional adjustment for some relations. One of these relations regards the simplified calculation of inelastic displacements and represents the main objective of this article. The paper uses dynamic nonlinear analysis performed on single degree of freedom systems as the main investigation tool. For all the analyses, two hysteretic models are used: Takeda and kinematic hardening. In the beginning, the paper presents the influence of overstrength on inelastic displacements for several behaviour factors. Next, the overstrength-period diagram is proposed based on a case study. Finally, the spectrum of $\mathrm{c}$, the ratio between inelastic and elastic displacement is calculated. The main result of the study is the simplified equations proposed for $\mathrm{c}$ factor, relations that can be successfully used in current design.
\end{abstract}

Keywords: inelastic displacement, behaviour factor, overstrength, elastic displacement

\section{Introduction}

The current Romanian seismic design code P100 is in a process of assimilation of the prescriptions of the equivalent European norm, EN1998-1.

One of those provisions relate to the determination of inelastic displacements of structures in a simplified way, using displacements of elastic analysis. The EN1998-1 does not directly specify a relationship between the elastic and inelastic displacement, but a relationship between behaviour factors and ductility. However, given the specific characteristics of Vrancea earthquakes, the relationship cannot be directly assimilated. In the new edition of P100, an improvement is attempted by considering the q-factor.

Moreover, similarly to EN-1998-1, the Romanian code will propose a relationship between the displacement requirement and the necessary reinforcement, as an alternative to using the minimum constructive reinforcement provided for the critical zone.

Considering all the above, the aim of the article is to present the hypotheses that form the basis of evaluating the elastic displacement amplification factor and to propose an accurate enough formulation depending on the q-factor.

According to P100/2006 the relationship between the elastic and inelastic displacements is given by:

$$
c=\frac{d_{i n}}{d_{e l}} c=\frac{d_{i n}}{d_{e l}}
$$

The coefficient $c$ does not depend on $q$, it only depends on the corner period $T c$ and the fundamental period of the structure, $T$ :

$$
c=3-2.5 \frac{T}{T_{c}} c=3-2.5 \frac{T}{T_{c}}
$$


It is noted that the value of the coefficient $c$ is limited to 1 for long periods and to 2 for the short ones. Also, the coefficient takes into account the overstrength of structures. EN-1998-1 does not specify an obvious relationship between the elastic and inelastic displacements. However, the following relationship is given between ductility and the real reduction factor:

$$
\mu=\left\{\begin{array}{c}
1+\left(q_{\mu}-1\right) \frac{T_{c}}{T}, \text { if } \mathrm{T}<\mathrm{T}_{\mathrm{c}} \\
q_{\mu}, \text { if } \mathrm{T}>\mathrm{T}_{\mathrm{c}}
\end{array}\right.
$$

The commentary to EN-1998-1 specifies that the q-factors include an overstrength factor of 1.5. Therefore, in the current design, relationship (3) will be applied by replacing $\mathrm{q}_{\mu}$ with $\mathrm{q} / 1.5$. Relationship (1) may be written according to the ductility factor as:

$$
c=\frac{d_{i n}}{d_{e l}}=\frac{\mu \cdot d_{p l}}{d_{e l}}=\frac{\mu}{\frac{d_{e l}}{d_{p l}}}=\frac{\mu}{\frac{F_{e l}}{F_{p l}}}=\frac{\mu}{q_{\mu}}=\frac{\mu}{q} \cdot q_{o v s}
$$

\section{Influence of overstrength on inelastic displacement}

In order to determine the influence of overstrength on the inelastic displacement, a set of 40 accelerograms was considered. The accelerograms are compatible with the acceleration elastic spectrum of Vrancea source, having the corner periods $0.7 \mathrm{~s}, 1 \mathrm{~s}$ and $1.6 \mathrm{~s}$. The analysed periods range between $0.1 \mathrm{~s}$ to $3 \mathrm{~s}$ with a $0.05 \mathrm{~s}$ increment. For each period, each accelerogram was scaled so that the elastic spectrum of the artificial accelerogram should coincide with the corresponding elastic spectrum of the ground acceleration equal to $0.20 \mathrm{~g}$.

For the q-factor, a total of 6 levels were considered, namely: level 2 (quasielastic), level 3 (minimum for high ductility class - value recommended for central core structures), level 4, level 5, level 6 and level 7 (approximated value recommended for frame structures). For each qfactor, the following overstrength range was considered: 1, 1.5, 2, 2.5 and 3.

For each period, the normalized plastic force was evaluated:

$$
f_{p l}=\frac{S_{a}(T)}{q_{\mu}}=\frac{S_{a}(T)}{q} \cdot q_{\text {ovs }}
$$

The analysis was performed on single degree of freedom systems. Two hysteretic models were considered, namely the Takeda model and the bilinear model with kinematic hardening. The post-elastic stiffness is 5\% from the elastic one, for both models. Unloading stiffness and strength degradation were not considered. The critical damping ratio was considered $5 \%$, mass proportional.

The computation of the $\mathrm{c}$ factor has the following steps:

a) Propose a q factor;

b) Propose a level of the overstrength factor, qovs;

c) Determine system strength based on equation (5);

d) Compute displacement spectra for all the accelerograms and mean displacement spectrum;

e) Calculate the value of the $\mathrm{c}$ factor using relation (4). For each period, inelastic displacement is the value of the mean displacement spectrum, while elastic displacement is calculated based on the relation between displacement spectrum and pseudo-acceleration spectrum.

The calculations are performed for each overstrength factor and each behaviour factor. 
The figures below represent the variation of c spectrum. They were determined by means of a nonlinear dynamic analysis for all the 40 accelerograms considered. The dashed green line represents the relationship used in P100 and the dotted purple line represents the values of the c coefficient evaluated with the Vidic-Fajfar relationship in EN-1998-1, considering an overstrength of 1.5 for the whole period range $(q \mu=q / 1.5)$.

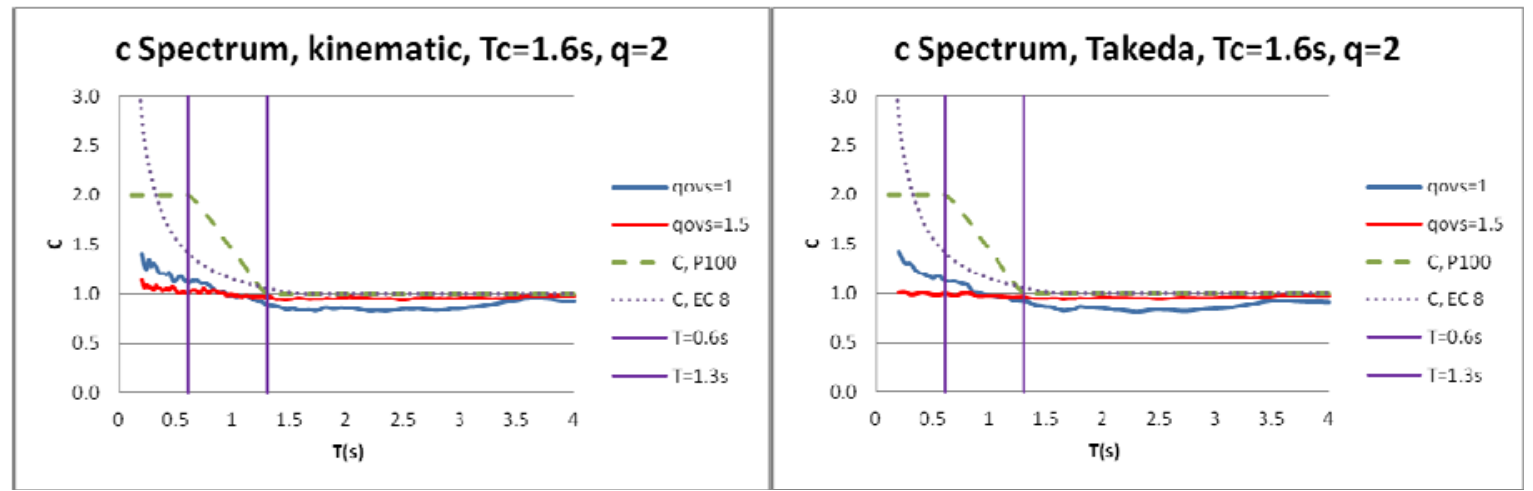

Fig.1 - c Spectrum, $\mathrm{T}_{\mathrm{c}}=1.6 \mathrm{~s}, \mathrm{q}=2$

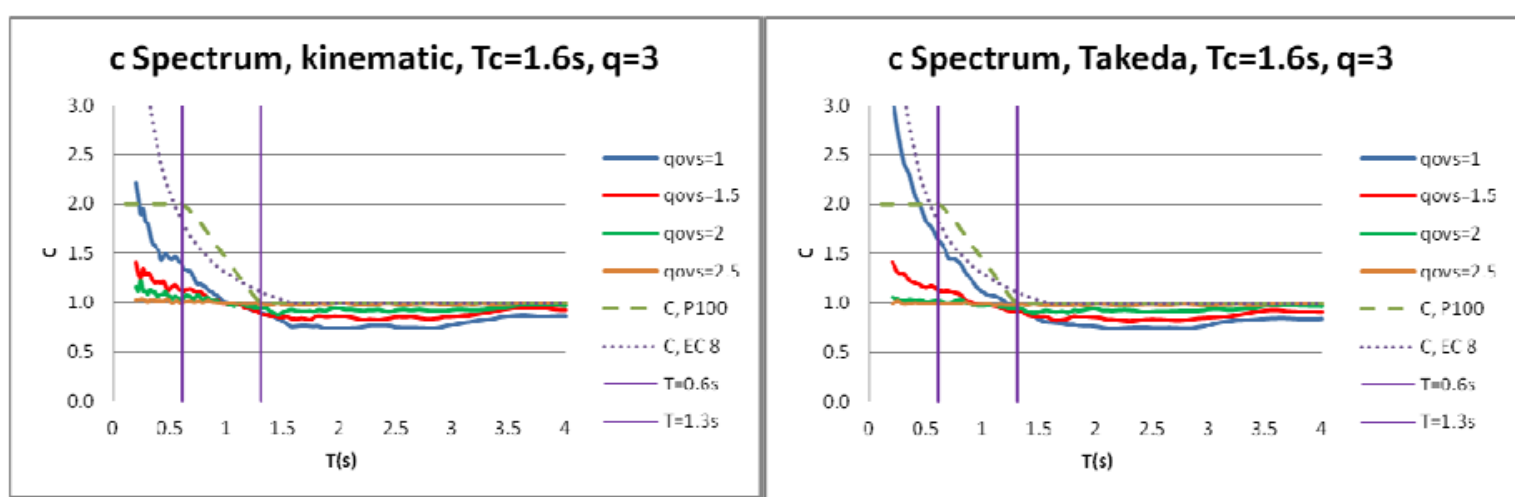

Fig.2 - c Spectrum, $\mathrm{Tc}=1.6 \mathrm{~s}, \mathrm{q}=3$

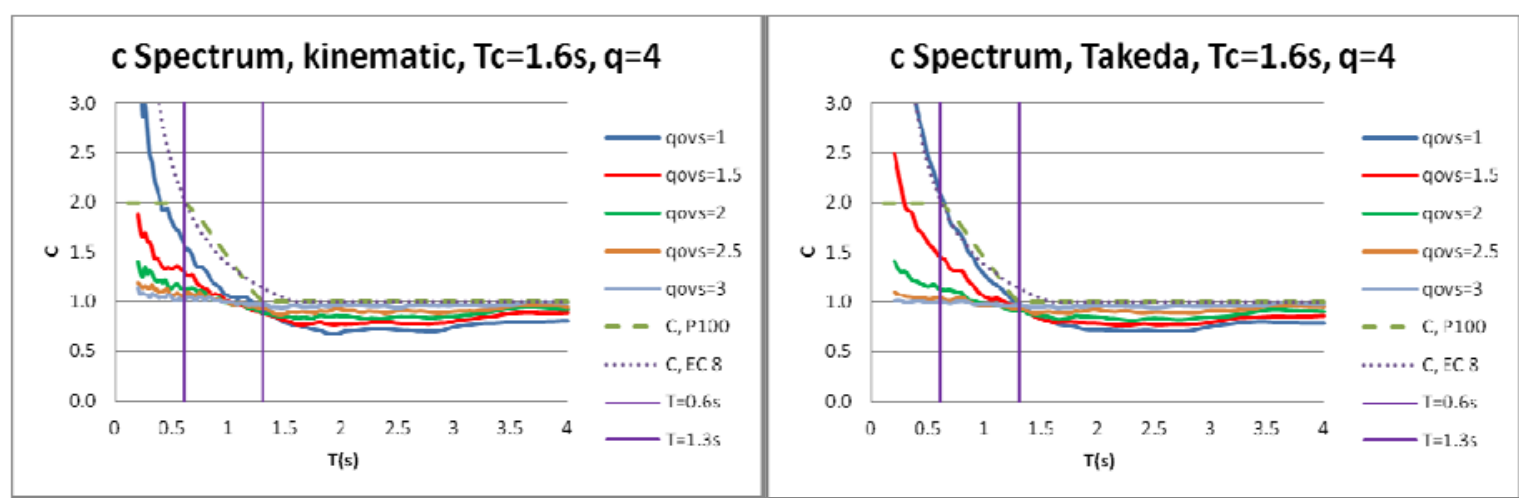

Fig.3 - c Spectrum, $T c=1.6 s, q=4$
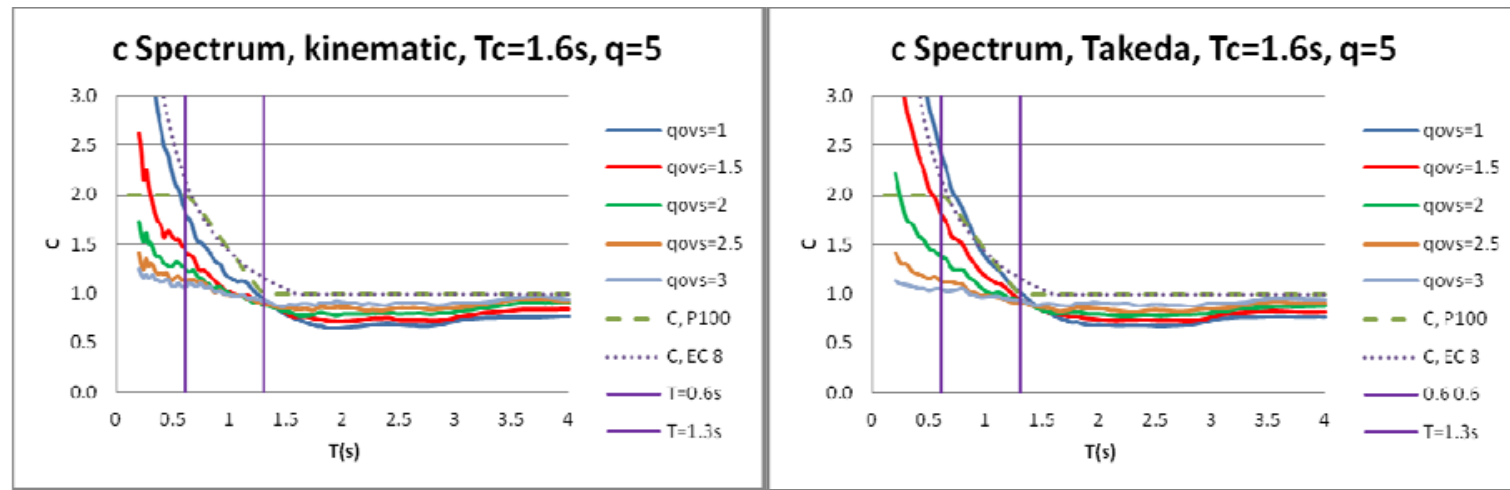

Fig.4 - c Spectrum, Tc=1.6s, q=5 


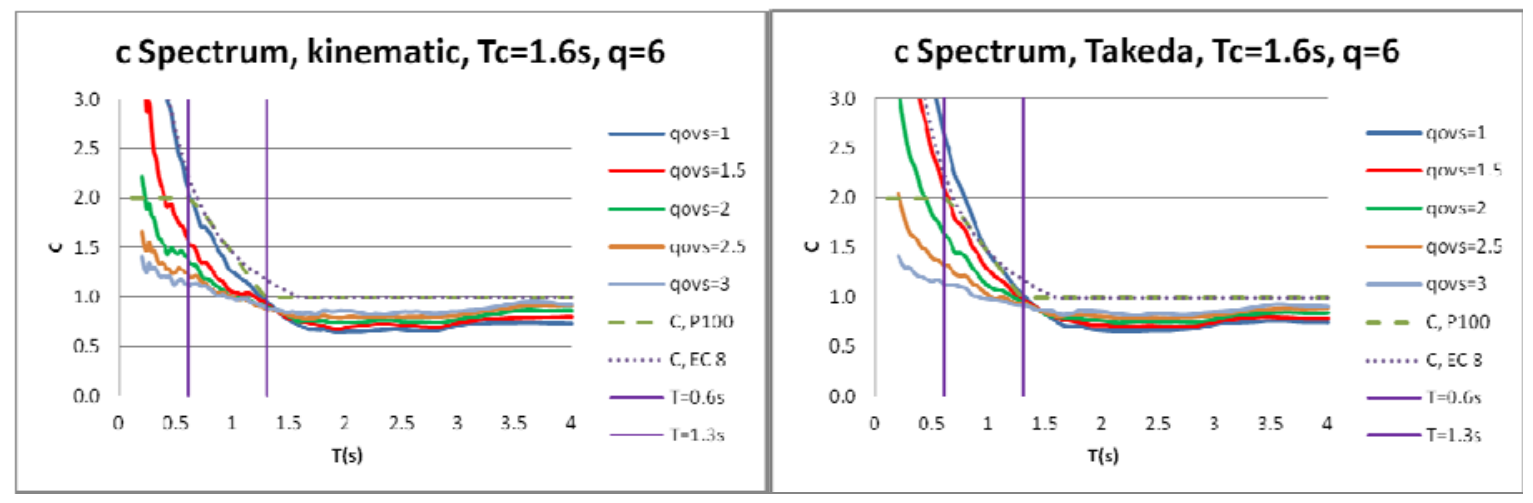

Fig.5 - c Spectrum, Tc=1.6s, q=6
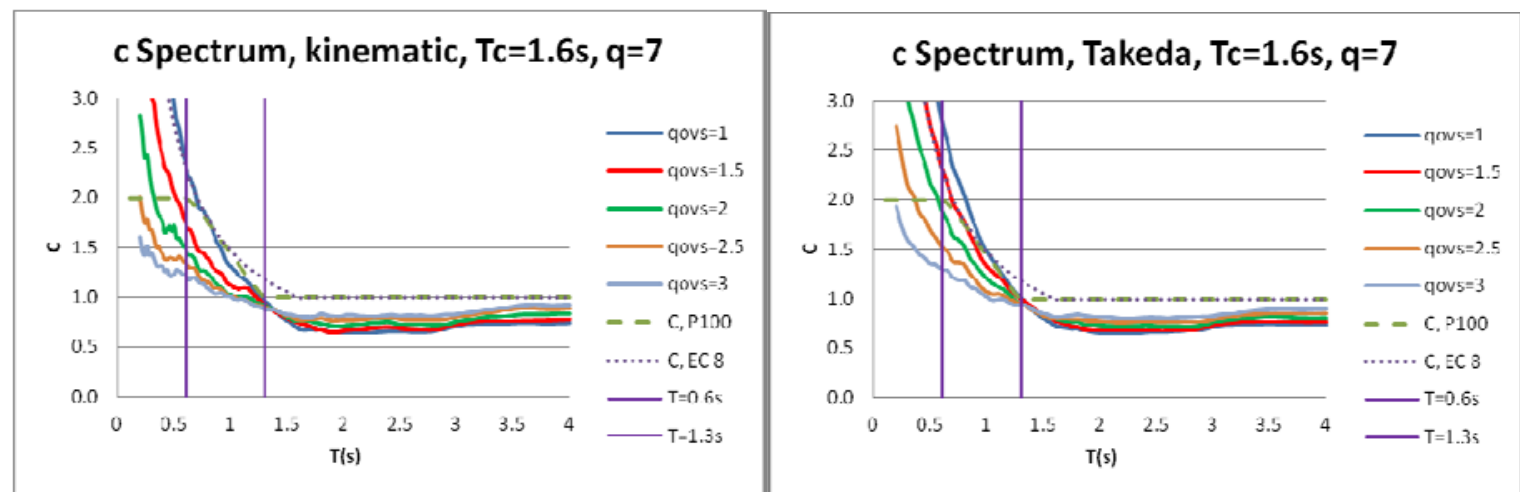

Fig.6 - c Spectrum, Tc=1.6s, q=7

By analysing the spectra a number of conclusions can be drawn.

a) The $c$ factor is less than one for periods above the corner period, no matter the q-factor and overstrength. However, the overstrength becomes significant for short periods and high overstrength, as can be noted from figures 1 to 6 .

b) The relationship used in P100 overestimates the inelastic displacement for medium and long periods, while for short periods the relationship underestimates the inelastic displacement in a few cases.

c) The similar relationship used in EN-1998 gives satisfactory results for long and medium periods, but overestimates significantly the inelastic displacements for short periods and small qfactors.

In conclusion, the overstrength is the key parameter in determining the inelastic displacement.

\section{Structural overstrength}

The overstrength is one of the most important parameters in the evaluation of the inelastic displacement. The relationship between ductility and the q-factor given in EN-1998 is determined on single degree of freedom systems without taking into account the overstrength. However, in the Designers Guide to EN-1998-1 specifies that the relationship should be used with a q-factor reduced by 1.5 in order to evaluate the ductility. This approach is on the safe side, the obtained displacement being higher than the ones resulted from the nonlinear dynamic analysis, as shown in the above chapter.

Overstrength has two main sources: material overstrength and structural redundancy overstrength. The main factor on which it depends is the fundamental period of the structure, 
because it is expected that rigid structures have significant dimensions of the main structural elements. These dimensions along with the minimum design prescription generally give a higher strength than the strength provided by the code. The main issue to be tackled is to determine the dependence of the overstrength on the period.

In order to consistently solve this issue a set of structures with different fundamentals periods should be considered. These should be designed for high and medium ductility class and analysed by means of the nonlinear static analysis.

However, even in this case the results have a degree of uncertainty due to the approximation of the fundamental period, nonlinearity modelling, etc. Therefore, to establish an order of magnitude of overstrength for short periods, a case study will be considered analysing a low height reinforced concrete structure.

For the analysis, a two span frame was considered. The loads were evaluated considering that the frame was part of a spatial structure with equal span and bay of 4 meters and floor height of 3 meters. The equivalent load on the beams was determined from a distributed load of $13 \mathrm{kN} / \mathrm{m} 2 \mathrm{in}$ the fundamental combination.

The structure was considered to be located in Bucharest, ground acceleration ag $=0.24 \mathrm{~g}$ and corner period $\mathrm{Tc}=1.6 \mathrm{~s}$. The seismic design was performed according to P100 using the equivalent static load method and a q-factor of 6.75 , corresponding to reinforced concrete framed structures. The resulting frame reinforcement is presented in fig. 7.

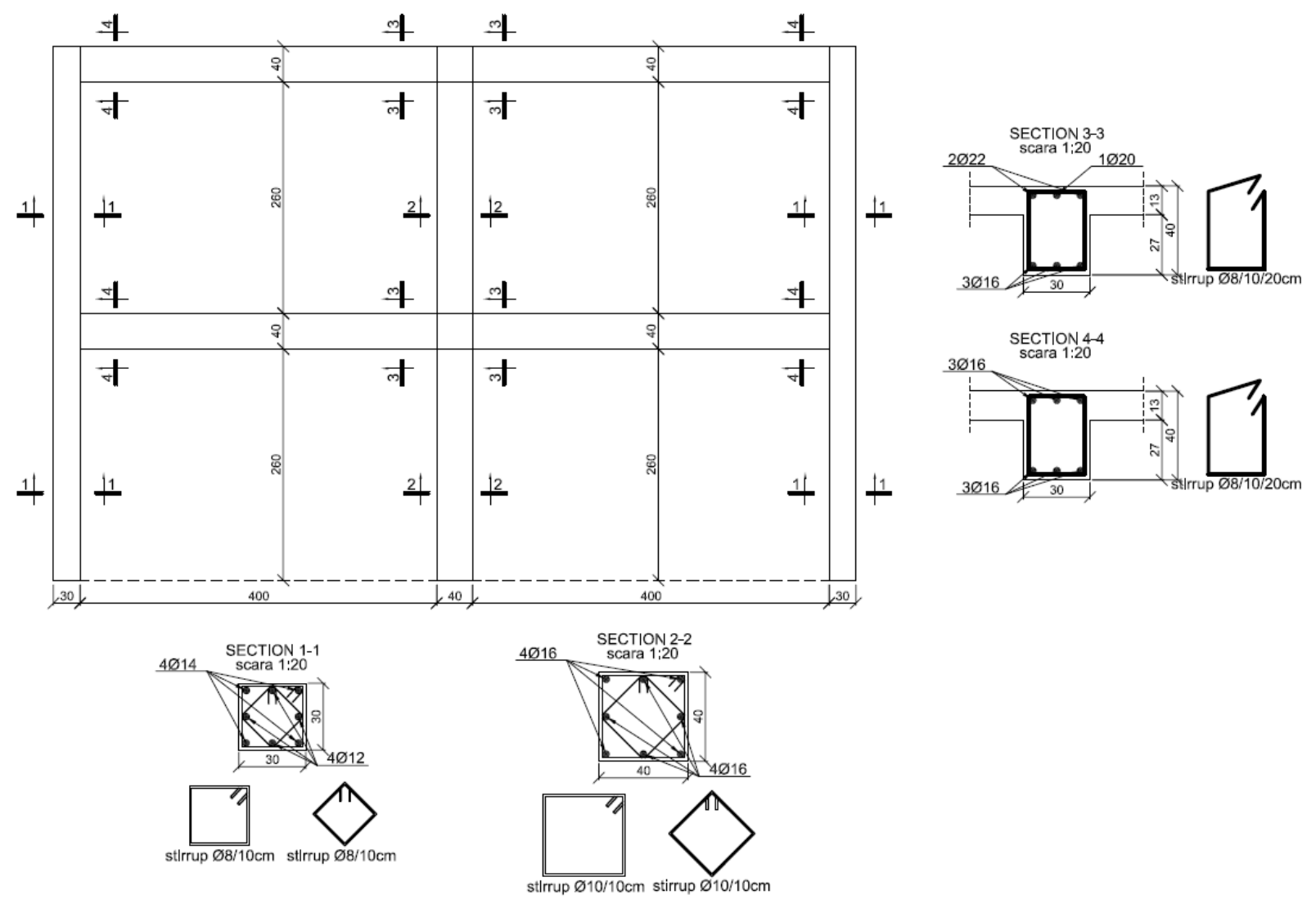

Fig. 7 - Reinforcement of the analysed frame

The fundamental period of the frame resulted $0.45 \mathrm{~s}$, corresponding to the cracked state of concrete $\left(\mathrm{Eb} \mathrm{b}^{\prime}=0.5 \mathrm{~Eb}\right)$.

The angular drifts were $1.6 \%<2.5 \%$ for ULS and $0.4 \%<0.5 \%$ for SLS. It can be observed that the code requirements are fulfilled with high safety factors.

Figure 8 presents the pushover curve corresponding to the fundamental mode of vibration. 


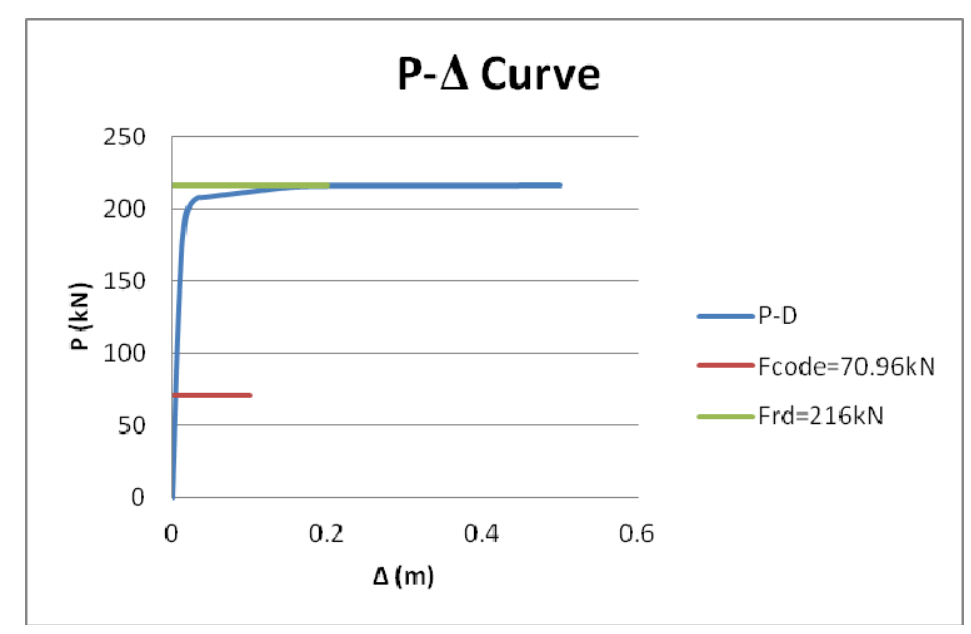

Fig. 8 - Pushover curve of the structure

$$
q_{\text {ovs }}=\frac{F_{c a p}}{F_{\text {cod }}}=3.04
$$

In conclusion, the study shows that for structures with short vibration periods designed according to P100, the structural overstrength is very high. The author proposes the diagram in figure 9 for redundant structures. A limiting period of $\mathrm{T}=0,2 \mathrm{~s}$ for $\mathrm{q}_{\mathrm{ovs}}=\mathrm{q} / 2$ was considered.

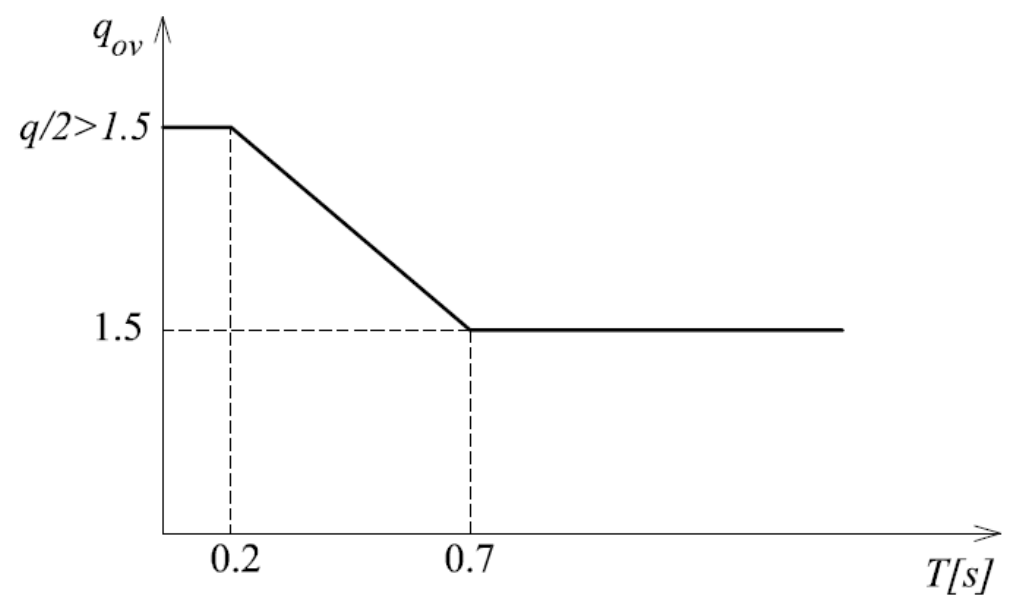

Fig. 9 - $\mathrm{q}_{\text {ovs }}$ diagram, redundant structures.

\section{Amplification factor (results)}

The nonlinear analysis results have been interpreted and the representation from fig. 10, fig. 11, fig. 12. It can be noticed that the values of the amplification factor depend significantly on the qfactor for short periods. This dependence is not so obvious for an intermediary period range centred on $0.8 \mathrm{Tc}$. This is the most uncertain range of the analysis. As expected, for small values of the q-factor, the $c$ coefficient is equal to one. For these reasons, a relationship depending only on the fundamental period of the structure and on the corner period for the amplification factor is proposed for the intermediary range. The upper limit of the amplification factor is a value depending on both the q-factor and on the corner period whereas the lower limit is 1 .

The following formula is proposed for reinforced concrete structures:

$$
1<c=3-2.3 \cdot \frac{T}{T_{c}}<\frac{\sqrt{T_{c} \cdot q}}{1.7} 1<c=3-2.3 \cdot \frac{T}{T_{c}}<\frac{\sqrt{T_{c} \cdot q}}{1.7}
$$




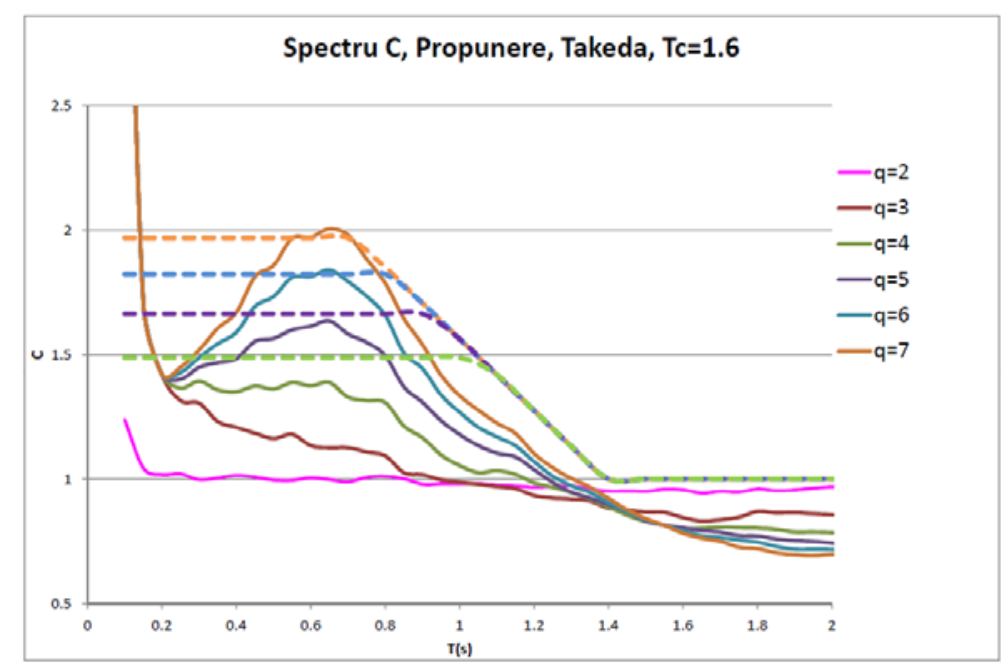

Fig. 10 - c Spectrum, Tc=1.6s, proposed formula for reinforced concrete structures (dashed line)

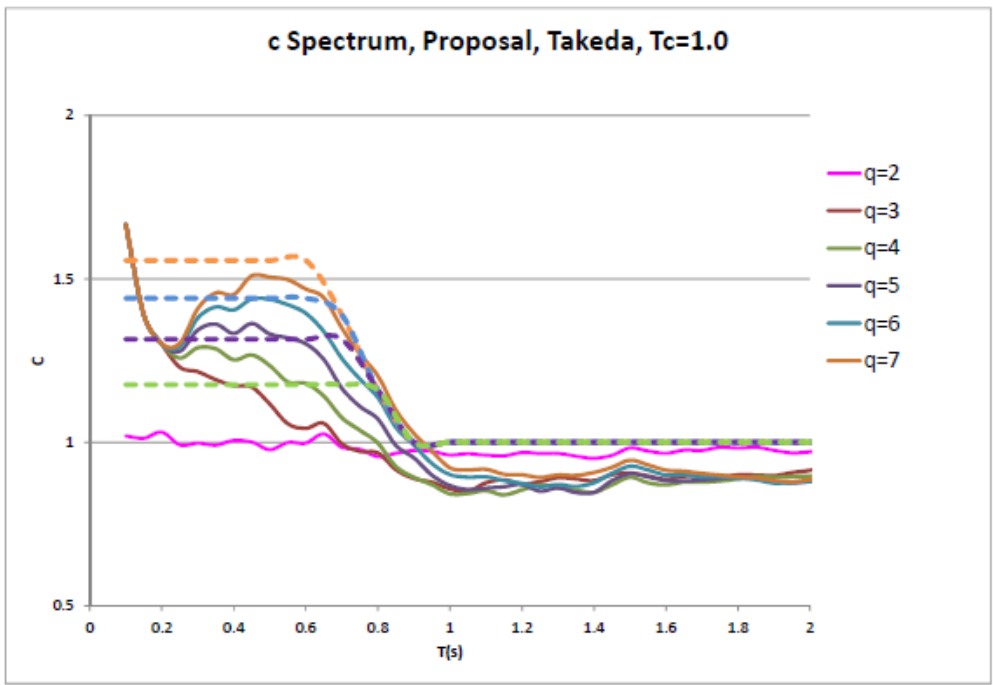

Fig. 11 - c Spectrum, Tc=1.0s, proposed formula for reinforced concrete structures (dashed line)

It is noticed that the proposed formulae approximates to a good degree the curves resulted from the nonlinear dynamic analysis for all corner periods. Also, the formula is on the safe side in most common cases. Furthermore the formula is not applicable to hall structures, where the overstrength differs significantly for the one considered in this article.

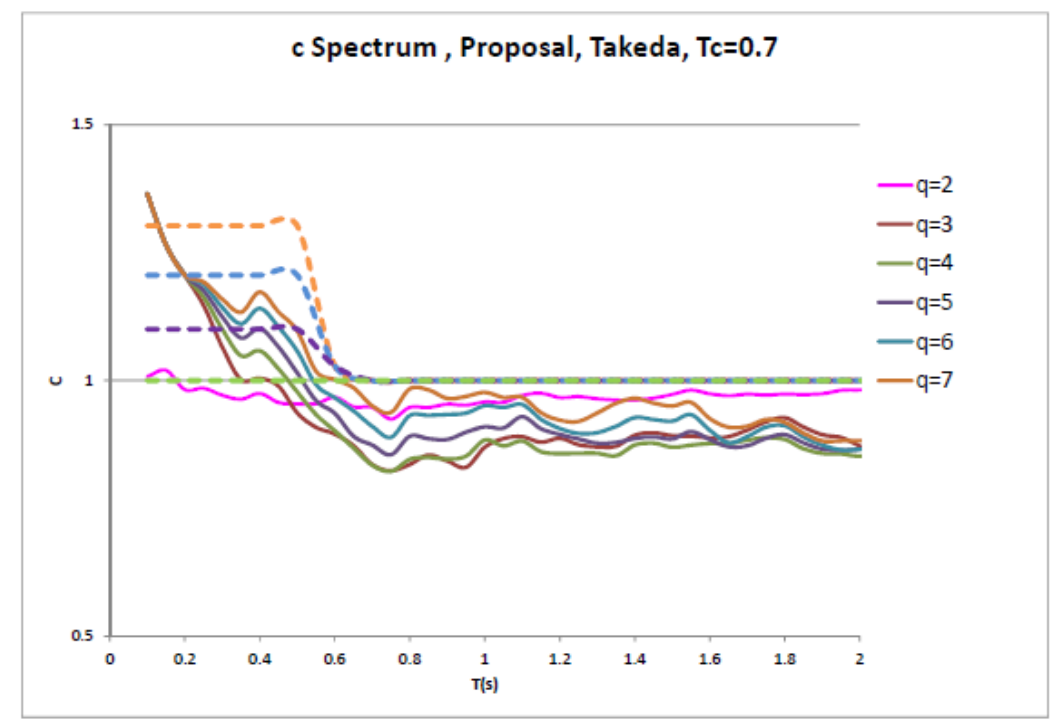

Fig. 12 - c Spectrum, Tc=0.7s, proposed formula for reinforced concrete structures (dashed line) 


\section{Conclusions}

The article aims to determine a simplified relationship between the inelastic and the elastic displacements. The influence of the most important parameter, that is the overstrength, is presented and analytical relationships are proposed for the evaluation of the inelastic displacement of reinforced concrete structures.

The present study improves the prescriptions given in the current seismic code P100/2006 by introducing an elastic displacement amplification factor dependent on the q-factor for reinforced concrete structures. It appears that the relationship used to evaluate the maximum displacements given in P100/2006 proves to be too "safe" for low rise structures which have significant overstrength.

The relationships given in EN 1998-1 are even "safer" for such structures. This implies that the resulting confinement reinforcement obtained based on the ductility requirement cannot be laid out in the element due to lack of space. This is the case when the displacement requirements are overestimated thus a more refined estimation is imperative.

Future research directions include performing overstrength analyses for different types of structures and different periods, including steel structures.

\section{References}

[1] P100.(2006) Seismic Design Code - Part 1 - Design Provisions for Buildings

[2] EN1998-1. Design of structures for earthquake resistance - Part 1: General Rules Seismic Action and Rules for Buildings

[3] Fardis, M., Carvalho, E., Elnashai, A., Faccioli, E., Pinto, P., Plumier, A., (2005). Designer's Guide to EN19981 and EN1998-5. Eurocode 8: Designer's Structures for Earthquake Resistance. General Rules Seismic Actions Design Rules for Buildings Foundations and Retaining Structures. Published by Thomas Telford Publishing. 